\title{
Sport for development in policy, practice and research
}

Sport has a lengthy history of servicing 'social development' objectives. The contemporary SfD movement is thus following a well-known tradition that includes the use of sport to support, for example, 'muscular Christianity' in the nineteenth century and diverse development aims in the twentieth (Beacom, 2007; Kidd, 2011; Darnell, 2012). The use of sport for these purposes has been underpinned by assumptions that sport can be beneficial to individuals and also to 'society' - by, for example, promoting inclusion, regulating undesirable behaviour or contributing to public health. From some perspectives, therefore, $\mathrm{SfD}$ in the twenty-first century might appear to be no more than 'old wine in new bottles' (Kidd, 2012). In practice, however, while the current SfD movement has strong associations with both its more distant historical legacy and its recent past, it combines these with unprecedented scale, complexity and ambitions. This amounts to a step change in expectations of what sport can deliver.

This chapter will explore the global development, growth and operation of contemporary SfD. We do this, in part, by reviewing existing research on SfD. In this way, the chapter fulfils two key purposes in developing our analysis through the book. First, the broad contextualization offered here situates subsequent chapters that seek to 'localize' global SfD. Second, our approach in the chapter also enables identification of themes and understandings emergent from the increasing academic research on $\mathrm{SfD}$ as well as exploration of the epistemological and methodological underpinnings of this research. To these ends, the chapter covers the global emergence of $\mathrm{SfD}$, the expectations that this attaches to sport as an agent of social change, the array of organizations and practices that operate within the SfD 'sector', and debates about the SfD 'evidence-base'. The final section of the chapter then considers our approach to localizing the global understandings of earlier sections. We explain the alignment of our research with the actor-orientated sociology of development advocated by Long (2001) and discuss how this may complement, challenge and extend existing knowledge on SfD. 


\section{Towards a global movement: the transformation of SfD}

Kidd $(2008,2012)$ has described SfD as an 'international movement' that by 2012 had rapidly progressed from being a 'new social movement' to a 'maturing one'. Identifying the precise point at which a new social movement emerges and then passes through significant phases in its evolution is never straightforward. Many, however, point to the establishment of the organization Olympic Aid (subsequently Right to Play) by the speed skater Johann Koss after the Lillehammer Winter Olympics of 1994 as a symbolic initial point of departure (e.g. Kidd, 2008; Young and Okada, 2014a).

Table 2 Exemplar global and local developments in the emergence of SfD

\begin{tabular}{cc}
\hline $\begin{array}{c}\text { UN and global actions contributing } \\
\text { to increasing global profile of SfD }\end{array}$ \\
\hline $1980 \mathrm{~s}$ & \\
$1990 \mathrm{~s} \quad \begin{array}{c}\text { Establishment of fundraising } \\
\text { organization Olympic Aid } \\
\text { (subsequently to become Right to } \\
\text { Play) (1994) }\end{array}$
\end{tabular}

Selection of 'localized' African

and Zambia-related developments

Initial Norwegian support and funding for sports development in Tanzania

Formation of MYSA (Kenya,1987)

Initial Norwegian support for NSCZ (1990)

Sport in Action and EduSport formally established in Zambia (1999)

2001 Appointment of Adolf Ogi as UN's first Special Advisor on Sport for Development and Peace

Zambian leadership and Norwegian support leads to instigation of KAO network

2002 Establishment in 2002 of the UN's Inter-Agency Task Force on Sport for Development and Peace

Establishment of a Right to Play National Office in Zambia

UK Sport joins KAO network and begins work with Zambian NGOs

2003 First International Conference on Sport and Development in Magglingen, Switzerland Publication of Sport for Development and Peace: Towards Achieving the Millennium Development Goals(UN InterAgency Task Force on Sport for Development and Peace, 2003) 
Table 2 Exemplar global and local developments in the emergence of SfD (Continued)

Selection of 'localized' African

$\mathrm{UN}$ and global actions contributing and Zambia-related developments to increasing global profile of SfD in $\mathrm{SfD}$

2004 UN establishment of SDPIWG

2005 Designation of International Year of Sport and Physical Education by the UN General Assembly

Establishment of UNOSDP

Second International Conference on Sport and Development in Magglingen, Switzerland

Commencement of IDEALS programme through partnership between UK Sport, British universities and Zambian NGOs

Commencement of II programme in Zambia through partnerships between Zambian NGOs, NSAs, government ministries, UNICEF, UK Sport and the British Council

2008 Publication of SDPIWG report 'Harnessing the Power of Sport for Development and Peace:

Recommendations to Governments' (SDPIWG, 2008a)

EduSport's Go Sisters programme receives funding from the DfID

Withdrawal of Right to Play from Zambia

Opening of Olympic Youth Development Centre in Lusaka, built with Olympic Solidarity funds

2015 Specific mention of the contribution of sport in the '2030 Agenda for Sustainable Development' (UN General Assembly, 2015) 
However, the most visible period of growth, in terms of both the international recognition and institutional status of $\mathrm{SfD}$, occurred in the opening years of the twenty-first century. During this time the UN raised the global status of SfD through a series of high-profile and formalized actions. The timeline of these global actions, summarized in Table 2, has been widely documented in academic literature (e.g. Beutler, 2008; Kidd, 2008; Levermore and Beacom, 2009a; Giulianotti, 2011a; Hartmann and Kwauk, 2011; Young and Okada, 2014a) - yet it represents only a very selective picture of the development of the SfD movement. Recognition of the emergence of specific SfD organizations and programmes in particular locations, some prior to the 1994 'starting point' of SfD, appears more disparately across different sources (e.g. Coalter, 2009; Cornelissen, 2011; Straume, 2012) and does include the local and indigenous NGOs that we have worked with in Zambia - Sport in Action and EduSport, which were formally established in 1999. The importance of the selected 'localized' developments presented in Table 2 is to indicate that the SfD movement is not necessarily or universally of recent origin nor is it solely the product of global North ${ }^{1}$ interests. Nevertheless, what has been transformed in recent years is the level of international prominence and support gained by SfD.

The UN's central role in international development has been instrumental in gaining recognition for sport within the wider global development system. This has been a process in which the lines between advocacy and analysis have often been blurred. International SfD specialists with academic, policy and practice backgrounds have come together to attend events such as the Magglingen conferences in Switzerland in 2003 and 2005, from which formal statements have been made endorsing the role of sport in achieving development. Further influential documents have been produced through similar collaboration under the auspices of the UN by the SDPIWG, whose formal remit is to 'promote the integration of sport within government development policies' (italics added; UN, n.d.). The Working Group's 'academic-advocacy' outputs include a set of literature reviews on the contributions of sport to development (SDPIWG, 2007), and the weighty 324-page report, 'Harnessing the Power of Sport for Development and Peace: Recommendations to Governments', authored by Right to Play on behalf of the wider SDPIWG (2008a). Both have been widely disseminated and cited, and alongside other UN publications, have come to 'represent the reproduction of the SDP discourse'2 (Hayhurst, 2009: 205). Further resolutions and policy documents that have subsequently emerged 
from the UN and other international organizations (e.g. Kay and Dudfield, 2013) have added to the recognition of SfD and its momentum (Hayhurst, 2009; Darnell, 2012).

As will be further examined in the next section with respect to the MDGs, SfD has become increasingly associated with global frameworks for international development. Darnell (2012) argues that this alignment was enabled by the association of SfD with the prevailing neo-liberal discourses dominant in international development policies and structures. For example, the SDPIWG (2006: 2) itself offered sport as 'a low-cost, high-impact tool to achieve broader development aims'. In this respect, development agencies had little to lose in incorporating sport and sporting organizations into international development frameworks and the proactive case for SfD was strongly voiced by advocates from this sector. In 'Harnessing the Power of Sport for Development and Peace' it was stated that sport's 'popularity transcends national, cultural, socioeconomic and political boundaries and can be invoked with success in virtually any community in the world' (SDPIWG, 2008a: 5). According to Darnell (2012), such a 'universality' discourse serves to depoliticize sport and, as such, make it a more amenable tool to be appropriated by international development agencies. Moreover, advocates positioned SfD as representing something of a new and alternative engagement approach which provided further traction at a time when mainstream development policies were under scrutiny for their limited success in reaching the world's poorest and most marginalized people (Levermore and Beacom, 2009a).

The 2003 Magglingen Declaration, which formally recognized sport as a contributor to the MDGs, was a significant milestone in the association of SfD with international development frameworks. The MDGs themselves were the first universally adopted global framework for development, and represented a new strategy for the international community. Where previously sport had held a widely recognized but seldom formalized status as an agent for social change, it was now officially associated with the most ambitious and high-profile global development agenda ever established. This elevated status had implications far beyond the relatively small number of sporting organizations and individuals who might become directly involved in MDG-related activities: it gave sport unprecedented standing as a potential vehicle for social change. With this came high visibility, quite specific and ambitious goals, and an exceptional level of scrutiny. In the next section we try to unpick the assumptions and expectations that this position placed upon sport. 


\section{Delivering the international development agenda: sport as an agent of social change}

Since 2000, the MDGs have framed international development policies and agendas. The MDGs initially emerged from the Millennium Declaration in September 2000, signed by representatives of 189 countries including 147 heads of state and government, and were further endorsed by member states at the 2005 UN World Summit (UN General Assembly Resolution A/RES/60/). They identified separate goals and targets for eight particular policy areas (Box 1), but these were meant to be seen as an interrelated whole. The MDGs were also intended to

\section{Box 1: Contribution of sport to the MDGs}

Text of the factsheet published by the UNOSDP, February 2010

The Millennium Development Goals (MDGs) were established at the UN Millennium Summit in September 2000. The eight MDGs aim to eradicate or reduce poverty, hunger, child mortality and disease, and to promote education, maternal health, gender equality, environmental sustainability and global partnerships. The target date for achieving the MDGs is 2015.

Sport has been recognised as a viable and practical tool to assist in the achievement of the MDGs. While sport does not have the capacity to tackle solely the MDGs, it can be very effective when part of a broad, holistic approach to addressing the MDGs.

\section{MDG1: ERADICATE EXTREME POVERTY AND HUNGER}

- Participants, volunteers and coaches acquire transferable life skills which increase their employability

- Vulnerable individuals are connected to community services and supports through sport-based outreach programs

- Sport programs and sport equipment production provide jobs and skills development

- Sport can help prevent diseases that impede people from working and impose health care costs on individuals and communities

- Sport can help reduce stigma and increase self-esteem, self-confidence and social skills, leading to increased employability 
Box 1: Contribution of sport to the MDGs (Continued)

\section{MDG2: ACHIEVE UNIVERSAL PRIMARY EDUCATION}

- School sport programs motivate children to enrol in and attend school and can help improve academic achievement

- Sport-based community education programs provide alternative education opportunities for children who cannot attend school

- Sport can help erode stigma preventing children with disabilities from attending school

\section{MDG3: PROMOTE GENDER EQUALITY AND EMPOWER WOMEN}

- Sport helps improve female physical and mental health and offers opportunities for social interaction and friendship

- Sport participation leads to increased self-esteem, self-confidence, and enhanced sense of control over one's body

- Girls and women access leadership opportunities and experience

- Sport can cause positive shifts in gender norms that afford girls and women greater safety and control over their lives

- Women and girls with disabilities are empowered by sport-based opportunities to acquire health information, skills, social networks, and leadership experience

\section{MDG4: REDUCE CHILD MORTALITY}

- Sport can be used to educate and deliver health information to young mothers, resulting in healthier children

- Increased physical fitness improves children's resistance to some diseases

- Sport can help reduce the rate of higher-risk adolescent pregnancies

- Sport-based vaccination and prevention campaigns help reduce child deaths and disability from measles, malaria and polio

- Inclusive sport programs help lower the likelihood of infanticide by promoting greater acceptance of children with disabilities 
Box 1: Contribution of sport to the MDGs (Continued) MDG5: IMPROVE MATERNAL HEALTH

- Sport for health programs offer girls and women greater access to reproductive health information and services

- Increased fitness levels help speed post-natal recovery

\section{MDG6: COMBAT HIV AND AIDS, MALARIA, AND OTHER DISEASES}

- Sport programs can be used to reduce stigma and increase social and economic integration of people living with HIV and AIDS

- Sport programs are associated with lower rates of health risk behaviour that contributes to HIV infection

- Programs providing HIV prevention education and empowerment can further reduce HIV infection rates

- Sport can be used to increase measles, polio and other vaccination rates

- Involvement of celebrity athletes and use of mass sport events can increase reach and impact of malaria, tuberculosis and other education and prevention campaigns

\section{MDG7: ENSURE ENVIRONMENTAL SUSTAINABILITY}

- Sport-based public education campaigns can raise awareness of importance of environmental protection and sustainability

- Sport-based social mobilization initiatives can enhance participation in community action to improve local environment

\section{MDG8: DEVELOP A GLOBAL PARTNERSHIP FOR DEVELOPMENT}

- Sport for Development and Peace efforts catalyze global partnerships and increase networking among governments, donors, NGOs and sport organizations worldwide 
be based upon a partnership between countries from the so-called global North and South, 'to create an environment - at the national and global levels alike which is conducive to development and the elimination of poverty' (UN, 2000).

The MDGs were neither the first nor the only shared targets that the international community had adopted to drive development. They were, however, the first integrated approach to development that set time-bound, quantified targets across multiple policy dimensions. They also gave global development an easily recognized identity that could be used to gain popular and political support worldwide, and thus had considerable symbolic significance. For sport, the MDGs were especially significant as they came to the fore at exactly the period when SfD formally entered the development framework. After the 2003 Magglingen Declaration, the incorporation of SfD into international development was repeatedly reinforced by citation of the MDGs in UN-sponsored SfD policy documents and other prominent publications. The broad scope of the Goals was reflected in the very wide-ranging statements about the contribution sport could make to them, often made or emphasized by those who have been identified as 'sports evangelists' (Coakley, 2011). Similarly, Hayhurst has shown that, by 2009, sport had been identified in various UN-sponsored SfD publications and policy documents as having capacity for:

Alleviating the negative aspects of poverty, achieving both community and individual health, facilitating healthy human development, building social capital, driving economic and community development, assisting youth-at-risk, fighting gender inequalities, promoting human rights, improving quality of life, reducing conflict between communities and nations by promoting peace, reaching individuals with disabilities, and fostering sport development. (Hayhurst, 2009: 213)

In practice, sport was most commonly associated with the goals that focused on individual and social change, especially (i) combatting HIV/AIDS, primarily through education about the virus and disease (part of MDG6); (ii) empowering women and girls (MDG3); and (iii) promoting education (MDG2) and wider youth development. Addressing these particular Goals was also expected to make some contributions to others - for example, gender empowerment though sport could overlap with MDGs 4 and 5, which both addressed gendered child and maternal health issues. Addressing both education and health was also central to the overarching aim of reducing world poverty (MDG1). In fact, the short factsheet published by the UNOSDP in February 2010 (Box 1) sought to claim that sport could support all eight of the MDGs, including MDG7 (ensure environmental sustainability) and MDG8 (develop a global partnership for development). 
The connection with HIV/AIDS was arguably the most significant to the modern international SfD movement. It is also the context within which the starting point for SfD was most easily identifiable, with sport-based programmes emerging in response to the HIV/AIDS pandemic which dominated the development agenda in Africa from the mid-1990s. The first efforts in sport to develop specific responses to HIV/AIDS came from individual SfD organizations; as explored further in the next chapter, it was Zambia's EduSport Foundation, itself founded in 1999, which in 2001 helped to initiate the international KAO Network. The association of sport with HIV/AIDS thus predated the formalization of the SfD movement over the following years, but gave strong momentum to it (Coalter, 2009). (It is worth noting that in contrast, there are no such readily identifiable global starting points for the emergence of SfD programmes using sport to address youth development or gender empowerment, both of which were long-established areas of activity prior to their incorporation into the international development agenda.)

Nevertheless, as demonstrated by Box 1, the claims made for the contributions of sport to HIV/AIDS, youth development and gender empowerment as well as other MDGs were both diverse and highly ambitious. The question of how sport may (or may not) contribute to development aspirations and debates is a central one within $\mathrm{SfD}$, and later sections of the chapter consider the state of evidence regarding its potential development impacts. First, however, it addresses another area of uncertainty - the scale and characteristics of organizations that can be considered to make up the ascribed 'SfD movement'.

\section{Recognizing the (organizational) field of SfD}

The increasing recognition - and expectations - of SfD has led to a rapid increase in the number and types of organizations involved in the field. Hayhurst (2009: 206) describes 'a wide variety of entities, such as UN agencies, faithbased groups, universities, academics, international organizations, governments, sport federations, and NGOs' as being involved in SfD; to these might be added schools, community groups, Olympic committees, commercial-sector organizations and a whole range of policy interests, each with its own network of actors coalescing around issues such as youth, gender, health and education. Given such a range of organizations, it is unsurprising that Kidd (2011: 604) observes, 'a tremendous diversity of purposes, methodologies, actual activities [and] levels of intervention' are associated with SfD. In attempting to bring some classificatory order to this diversity, both Akindes and Kirwan (2009) and Giulianotti (2011a) 
offer categorizations of types of SfD organizations, both of which are based on traditional demarcations between organizations from the public, private and civil-society $^{3}$ sectors. Giulianotti (2011a) adds some complexity by adding the policy orientation of organizations into his classification. However, the empirical basis of Akindes and Kirwan's (2009) classification is unclear and Giulianotti's (2011a) is limited by being drawn from investigations in Europe, Asia and the Middle East alone, thus omitting the high level of SfD activity in Africa.

More generally, it can be argued that the sector remains under-documented and, similarly, this has prompted calls from both practitioner and academic sectors to 'map' organizations involved with SfD (e.g. Kidd, 2011; Lyras and Welty Peachey, 2011). For example, Donnelly et al. (2011: 593) advocate that, 'At the very least it is necessary to collect detailed data regarding the scope of SDP, the numbers involved, and the depth and breadth of involvement.' However, the underpinning rationale for these mapping or classificatory efforts is not always clear and McFarlane (2006, cited in Levermore and Beacom, 2012) warns of the colonial history of Northern efforts to map and classify development 'territories'.

Nonetheless, the need for basic information about the SfD field has led to various efforts to quantify it and common reference has been made to the number of organizations that have self-registered with the International Platform on Sport and Development (e.g. Levermore, 2009; Darnell and Black, 2011; Mansfield, 2013). These figures have consistently shown growth in the number of SfD organizations, nearly doubling in three years, from the 166 reported by Kidd in 2008 to 295 reported by Hartmann and Kwauk in 2011. The meaning of these figures is, however, very uncertain - the rise may well signify a genuine increase in the size of the sector, but might also indicate an increased tendency among certain types of organizations to register with the Platform. This uncertainty means that there are no grounds for judging whether the increase in registrations with the Platform corresponds with a proportionate change in the number of SfD organizations as a whole. Moreover, Donnelly et al. (2011: 593) suggest 'there is every reason to suspect that these [organizations registered with the Platform] represent the thin end of the wedge', and data on SfD organizations that have not registered with the Platform or any similar international body is scarce and piecemeal. Nevertheless, what does emerge from this very limited evidence is a picture of an SfD sector that is 'loose knit' (Darnell, 2012: 86), diverse, diffuse and populated by numerous organizations - at least in some regions and localities.

Leading on from this last point, a further uncertainty facing those hoping to demarcate the field of SfD is the varied approaches that have been adopted 
to defining the geographical and national/international character of SfD. While Darnell (2012: 2) identifies the global South as 'the quintessential site of development', other authors do not differentiate between SfD programmes that are implemented in the global North and the global South (Coakley, 2011; Lyras and Welty Peachey, 2011). Although there are clearly recognized dangers of reinforcing a binary between the global North and global South (McEwan, 2009), there can be greater risk in failing to ascertain whether particular organizations, especially NGOs, operate internationally from the global North, transnationally across locations in the global South or in a particular locality in the global South.

These issues have particular importance when SfD is defined primarily in the context and in terms of international development agendas. As we have identified, the period of high-profile growth for the SfD movement has been very closely associated with prominent milestones in international development policy. Although the MDGs addressed universal social problems, their primary focus was unquestionably the poorest citizens in the poorest countries - traditionally, the 'recipients' of international aid from the global North. Many accounts of SfD align with this, focusing only on international SfD activity that has been initiated by organizations from the global North (e.g. Giulianotti, 2004; Levermore and Beacom, 2009a; Jarvie, 2011; Darnell, 2012).

This 'sport as international development' form of $\mathrm{SfD}$ can involve a variety of governmental agencies, private companies and NGOs from the global North. These organizations can work unilaterally or in collaboration in 'international' programmes, and this can produce a messy structure. Within Northern governments, Hayhurst (2009) identifies that different ministries (such as sport, education or international development) may have responsibility for $\mathrm{SfD}$, which, she suggests, may contribute to ambiguity in terms of input into relevant policies. In addition, governmental agencies from different countries may also play a role in $\mathrm{SfD}$ in particular Southern countries, as is explored in Chapter 2 in respect of both British and Norwegian governmental agencies working in Zambia. Diverse international NGOs are also involved, including those such as Right to Play and KAO which are specifically orientated towards $\mathrm{SfD}$, but also several significant international sporting governing bodies, such as FIFA, which are not primarily concerned with SfD but have extended their work to include it. Finally, the involvement of transnational private-sector businesses in SfD, often for purposes of corporate social responsibility, has increasingly been highlighted by authors from North America (e.g. Huish, 2011; Donnelly et al., 2011) or those with a specific interest in this area (e.g. Levermore, 2011; Banda and Gultresa, 2015). 
A range of civil society organizations in Southern countries are also recognized in the literature, although they have tended to feature less as objects of research than international agencies. The prominence of civil society organizations in SfD can be linked to broader neo-liberal international development policies that have sought over time to reduce the role of Southern states (Laird, 2007). Such NGOs and CBOs are diverse: in addition to those specifically focused on SfD they also include religious bodies, sports and interest groups, youth groups, and schools and higher education institutions. Authors have commonly recognized the organizational fragmentation amongst civil society organizations involved in $\mathrm{SfD}$ and the associated problems of competition, duplication and lack of co-ordination (e.g. Giulianotti, 2011b; Huish, 2011; Kidd, 2011). Such issues, which have rarely been studied in depth in the SfD literature, are explored here in Chapters 2 and 3.

A small number of these Southern civil society organizations have become familiar throughout the literature, including the MYSA in Kenya, the Magic Bus and the GOAL projects in India, and the two Zambian NGOs with which we have worked, Sport in Action and EduSport (e.g. Levermore, 2008; Kidd, 2008; Coalter, 2010a). However, as there has been a tendency to view these organizations primarily in their role as partners in international externally funded programmes, the local origins and orientation of these - and of other organizations that may not receive international funding - can be overlooked. Several authors have, however, emphasized the existence, importance and potential independence of local civil society organizations (Armstrong, 2004; Coalter, 2009; Kay, 2011). Armstrong's (2004) study of a network of football clubs in Liberia - which predates the surge of research in this area - emphasized the bottom-up, locally-led nature of its emergence and orientation towards development and peace. More recently, Fokwang (2009: 214) used a study of an indigenous SfD organization in Cameroon to 'demonstrate clearly that there exist groups and associations in developing countries that are not necessarily dependent on external development assistance'. These studies highlight the complexity and diversity which makes overall mapping of SfD so problematic.

The role or contribution of Southern governmental and private sector organizations in SfD also remains largely unexplored. Kidd (2008, 2012) has repeatedly called for greater (presumably Southern as well as Northern) governmental involvement in SfD. While Kidd's contributions suggest a lack of engagement in SfD by Southern governments, Njelesani (2011) provides an example of an intervention by the Zambian government into physical 
education as being within the broader scope of SfD policy. In an alternative but similarly Southern-orientated perspective, Cornelissen (2011) suggests that private sector involvement could reduce the dependence of SfD on international funding. The contributions of Njelesani (2011) and Cornelissen (2011) are relatively isolated examples, but they do emphasize the need to avoid overarching dismissals of the involvement of Southern governmental and private sector agencies in SfD. Instead, further research is needed to explore the involvement of such stakeholders in SfD in different Southern contexts.

A summary of ways in which the complex array of organizations and agencies in the SfD field may be classified is presented in Table 3. A common theme in analyses of specific and structural relationships between different $\mathrm{SfD}$ organizations and agencies is the extent to which they entail Northern hegemony and maintain Southern dependency. Many authors subscribe to the view that 'the sport-in-development movement tends to be dominated by a "vertical hierarchy" which affects donor-recipient and North-South relationships' (Nicholls, 2009: 158). Referring more narrowly to individual SfD programmes, Levermore (2009: 41) describes how their 'governance ... tend[s] to be dominated by the higher-income (Northern) countries'. Akindes and Kirwan (2009: 241) go further, suggesting that 'sport-in-development programmes must rely on

Table 3 Classificatory characteristics of organizations in the SfD field

\begin{tabular}{ll}
\hline \multicolumn{1}{c}{ Organizational characteristic } & Classificatory distinctions/dimensions \\
\hline Core organizational purpose & $\begin{array}{l}\text { Specific use of sport to contribute to } \\
\text { development } \\
\text { Sport with interest in SfD } \\
\text { Particular development agendas (to } \\
\text { which sport activities may directed) }\end{array}$ \\
& $\begin{array}{l}\text { Public/governmental } \\
\text { Civil society }\end{array}$ \\
& $\begin{array}{l}\text { Private } \\
\text { Sector }\end{array}$ \\
Primary/headquarters location & Global North \\
& Global South \\
Scope of geographical operation & Transnational \\
& Bilateral \\
& National \\
Community-based & Primarily international \\
Organizational and funding relationships & Primarily national and/or local \\
\hline
\end{tabular}


external sources of funding for their operations' (italics added). Beyond governance and funding, the literature also highlights other dimensions through which Northern influence is exerted. Darnell (2007), Black (2010) and Forde (2013) all examine aspects of power relations when staff, volunteers and 'athlete ambassadors' from the global North undertake SfD work in various contexts in the global South. Forde (2014) critiques the Northern construction the SfD curricula, and Hayhurst (2009: 217) further identifies knowledge transfer as another practice in which the global South is subjugated in a recipient relationship with organizations from the global North. Kay (2012) further highlights the significance of donor-imposed monitoring and evaluation systems in formalizing the 'accountability' of Southern partners to Northern funders. Approaches such as the widely used logic model framework can shape all aspects of programmes, from defining the formal objectives which they pursue, to specifying the measures against which 'performance' will be assessed.

In addressing these issues, $\mathrm{SfD}$ researchers have increasingly drawn on theoretical approaches associated with the wider development studies field. Several have adopted a postcolonial orientation (e.g. Darnell, 2007, 2012; Hayhurst, 2009; Black, 2010; Darnell and Hayhurst, 2011; Nicholls et al., 2011; Forde, 2013, 2014), with some also drawing on Gramscian and Foucauldian theories of power in their critiques of $\mathrm{SfD}$. As a result, one of the most significant themes in the $\mathrm{SfD}$ literature is the dominance attributed to global North-orientated agendas in the field, and the common association of this with the global influence of neo-liberal political economies. Some authors do credit Northern SfD stakeholders with reflexive recognition of power relations associated with their work (Giulianotti, 2011b; Darnell, 2012), but few writings provide specific examples of Southern agency or detailed analysis of how this occurs. Notable exceptions are Fokwang's (2009) previously mentioned study in Cameroon and both Guest's (2009) and Hasselgård and Straume's (2015) accounts of local stakeholders resisting and reorienting international SfD programmes in Angola and Zimbabwe respectively. In fact, Fokwang cites the activities of a Cameroonian $\mathrm{CBO}$ as being part of a 'not-so-unique trend ... of social actors self-manag[ing] their "development" process without recourse to local government, the state and powerful Western institutions' (2009: 215). As shall be argued further in the chapter, collective methodological limitations of $\mathrm{SfD}$ research mean that it is difficult to determine if the exceptionalism of Guest (2009), Fokwang (2009), and Straume and Hasselgård's (2014) contributions is more broadly reflective of genuine limitations of Southern agency or is merely indicative of the fact that such agency remains relatively unexplored. 


\section{Understanding and conceptualizing SfD practice}

As the previous discussion has shown, the $\mathrm{SfD}$ sector is characterized by diversity. SfD organizations have adopted a wide array of practical approaches, described by Cornelissen (2011: 506) as a 'fairly loose amalgam of different approaches that incorporates a diverse range of philosophies, practices, operational formats and programmes'. A significant number of case studies of individual SfD programmes have emerged over recent years (see edited collections by Gilbert and Bennett (2012), Schulenkorf and Adair (2014), and Young and Okada (2014b) for multiple examples). Research has also considered SfD practices associated with both sporting mega-events (e.g. Cornelissen, 2011; Levermore, 2011; Darnell, 2012) and 'grassroots, community-based sport' (Hayhurst, 2009: 206). This book is concerned with the latter, and in addressing this we begin by considering descriptive and normative conceptualizations of SfD practice that have been given prominence in the literature.

Perhaps the most often referenced and long-standing distinction that is applied to different forms of community-level sport utilizes the labels 'development of sport' (also frequently referred to as simply 'sports development') (Kidd, 2008) and development through sport' (our italics). 'Development of sport' or 'sports development' is generally used to describe efforts to develop the institution of sport itself, including increasing sport provision (e.g. facilities and human resources), improving sport practices (e.g. coaching standards and inclusive delivery) and - especially - raising sport participation and sport performance levels (Levermore and Beacom, 2009a). Although, as we shall see throughout the book, much SfD activity involves a degree of sport development - and often a very substantial one - the development of sport per se is not considered the sector's primary aim. Rather, it is 'development through sport' which is strongly associated with, and central to, the concept of SfD. SfD itself refers to the potential of sport to provide development outcomes both individually and collectively, with the latter ranging from impacts on immediate social networks such as families and communities, to wider structural impacts. In the case of gender empowerment, for example, an SfD programme might provide opportunities for individual young women to gain skills and confidence; encourage their families and influential community stakeholders to permit female participation in sport; and, beyond this, aim to contribute to a wider shift in restrictive gender attitudes. In theory, sport is therefore a potential vehicle for multiple forms of development, at multiple levels.

When considered in relation to practice, the approaches and intentions of sports development and sport for development are not necessarily as distinct as 
the above outline definitions suggest. Our own research in this book and that of a number of other scholars suggest that the two are often closely intertwined. In their interviews with representatives of seven Zambian SfD organizations, Njelesani et al. (2014) found that all also had a focus on the development of football skills and argued that this, in fact, might conflict with aspirations to use sport to address broader development goals. In contrast, Giulianotti (2011b: 56) concluded from his work elsewhere with SfD practitioners that development of sport and development through sport could be compatible, describing them as 'not always oppositional' and potentially 'mutually advantageous'. Similarly, Darby (2012) identifies some football academies in Ghana whose aspirations cross both development of and development through sport.

A further classificatory conceptualization oft cited in the literature is Coalter's (2007a, 2009, 2010b) distinction between 'sport-plus' and 'plus-sport' approaches to achieving development objectives. Of the two, plus-sport approaches are possibly the more clearly defined, as those that use sport as 'a type of "fly paper" to attract young people to programmes of education and training' (Coalter, 2010b: 298). In contrast, sport plus are described by Coalter as those that give 'primacy to the development of sustainable sports organizations, programmes and development pathways' (2009: 58). Coakley (2011: 314) points out, however, that the classificatory distinction between plus-sport and sport-plus approaches can sometimes be 'fuzzy' - a fact also recognized by Coalter himself who, with Taylor (Coalter, 2010c: 15), states that 'it is difficult to sustain a strict division between plus sport and sport plus'. In addition to the concern about conceptual clarity, the ongoing uncertainty about classificatory distinctions also raises questions about their value as largely heuristic devices proposed and employed by academics from the global North. In this regard, it is perhaps notable that the literature does not currently address the extent to which SfD practitioners recognize or value the suggested distinctions.

Moving beyond classificatory schema, further critiques emerge within the literature focused on SfD practices. This identifies the prevalence within SfD of a focus on the personal and social development of young people involved in sporting activities. Authors such as Spaaij, however, caution against the assumption that sport will somehow 'automatically' deliver such developmental outcomes, warning that:

The transformative capacity of sport-based interventions for disadvantaged or disaffected young people can only be realized within a personal and social development approach and not by merely offering sport activities. (Spaaij, 2012: 77) 
The approaches used in SfD to achieve personal and social development through sport have also been subject to criticism for their strong individual behaviourist' emphasis (Cole, 2006; Coakley, 2011; Hartmann and Kwauk, 2011). This is seen by many writers as a significant limitation of SfD when seeking to achieve developmental outcomes that also require structural socioeconomic change (e.g. Darnell, 2010a; Cornelissen, 2011; Coakley, 2011). Huish suggests that:

SDP projects that see sport as an independent means to prosperous development and individual empowerment are likely to encounter the well-known challenges of pursuing sweeping structural change through empowerment at the individual level. (Huish, 2011)

Certainly, there are fewer studies that identify or analyse sport-based programmes within which practices explicitly aligned with wider community development are recognized. What examples there are of community development approaches in the literature tend to be drawn more from post-conflict zones in specific geographical areas than other development scenarios (e.g. Gasser and Levinsen, 2004; Sugden, 2006; Kath and van Buuren, 2014). It is in respect of 'divided societies' that Sugden (2011) suggests a 'ripple effect' of impact, moving from individuals to broader aspects of society; while conceptually useful, it would be beneficial if there was further articulation and evidence of the processes by which any 'ripple effect' may work.

An increasing number of authors extend the critique of the individualist orientation of $\mathrm{SfD}$ practice and, in doing so, some associate this with the broader theme of neo-liberal and Northern hegemony. With SfD programmes operating in the global South, Coalter identifies:

[an] implicit assumption ... that young people living in [such] 'disadvantaged communities' are themselves deficient and in need of 'personal development' of the type that sport is presumed to deliver. (Coalter, 2010c: 22-23)

Coalter's (2013) subsequent publication of empirical research further questions the presumption of deficiency and it is also notable that there is a relative absence of debate in the literature regarding the relative disadvantage that exists within countries and communities in the global South (McEwan, 2009). Nevertheless, Hayhurst (2009), Darnell (2010a, 2012), Hartmann and Kwauk (2011), and Forde (2014) all present similar arguments that associate SfD with presumptions of deficiency and link this to neo-liberal ideologies that emphasize the 
responsibility of individuals for overcoming their own 'underdevelopment'. In a further generalization of this analysis, Darnell and Black suggest that:

[the] SDP movement [is] largely align[ed] with, and therefore may effectively strengthen, hegemonic relations by reinforcing dominant social and economic hierarchies and the neoliberal logic of competitive social and economic relations. (Darnell and Black, 2011: 369)

At this stage, two points should be made with regard to these arguments. First, attributions of neo-liberal (and thus Northern) hegemony within the SfD literature have largely been made with reference to empirical research undertaken with SfD stakeholders from the global North. Second, the analysis presented by authors does not necessarily preclude the possibility that SfD could be used to challenge hegemonic relations, as Levermore and Beacom suggest:

Sport can be viewed as a form of resistance, which might occasionally challenge dominant systems and processes, thereby redressing unequal power relations in limited instances. (Levermore and Beacom, 2009b: 253-254)

As such, Darnell (2012) begins to argue, albeit in a somewhat brief and largely theoretical way, how adopting a critical pedagogy approach within SfD could be used to achieve the structural transformation that Levermore and Beacom (2009b) propose. Initially developed by Brazilian educator Paulo Freire (1972), critical pedagogy combines education with critical theory to prompt marginalized individuals to consider the causes and influences of their social conditions. The development of 'critical consciousness' provides the basis for potential collective social action and ultimately transformation of circumstances.

Darnell's (2012) advocacy for critical pedagogy is one example of a broader normative strand of the literature on SfD practices. However, in contrast to Darnell (2012), this normative strand is dominated by contributions that adopt a 'rational policy solving' (Mosse, 2004: 642) approach to improving SfD. Such contributions commonly call for improved management and implementation of programmes, with Coalter (2009: 65) suggesting that SfD interventions 'require a clear, articulated understanding and evaluation of the conceptualization, design and delivery of a programme'. A number of the more recently emergent case studies in the SfD literature also address similarly orientated themes. For example, amongst such case studies, there are calls for greater precision in the specification of desired outcomes through improved understanding of local 
needs (e.g. Richards and Foster, 2014; Siefken et al., 2014) and the development of SfD curricula together with associated documentation (e.g. Weinberg and Rockenfeller; 2012; Gannett et al., 2014; Siefken et al., 2014). Mosse would classify such contributions within a recognized 'instrumental' strand of the wider development studies literature, which he critiques as offering 'simplifying models of change [through] detailed planning and management procedures' (Mosse, 2004: 641).

Perhaps the most extreme examples of this trend are suggested 'models of SfD' for which significant claims are made. To varying degrees, such models are underpinned by assumptions that it is possible to identify 'key elements required for effective sport-based social intervention' (Hartmann and Kwauk, 2011: 289) that are generalizable across different contexts. The most ambitious claims are those of Lyras and Welty Peachey (2011:324) who offer what they term a 'sportfor-development theory', which they claim 'provide[s] the essential features and structure to scientifically explain, describe, and predict how sport can effect social change. Like Hartmann and Kwauk, Lyras and Welty Peachey mainly draw on research conducted in the global North, yet are confident that their theory also 'offers a framework that is applicable in international, national, and community-based sport interventions' (2011: 324). Schulenkorf's (2012: 9) claim of his alternative sport-for-development framework as a suitable guide for the strategic management and evaluation of (inter)community development projects' is only slightly more circumspect.

While Lyras and Welty Peachey (2011) and Schulenkorf (2012) do suggest that there is room within their models for locally owned and culturally appropriate development, they remain vulnerable to Darnell's concern that there is:

[ongoing] misalignment between northern notions of the preferred or requisite tools for successful development versus local demands regarding what constitutes the appropriate skills, resources or opportunities for success. (Darnell, 2012: 96)

In particular, these models are representative of the 'positivist-based problemsolving orientation' that Darnell (2012: 40) and Nicholls et al. (2011) identify as prominent within the $\mathrm{SfD}$ sector more generally. Such an orientation tends to universalize $\mathrm{SfD}$ and underplay diversity and complexity within the sector. Some of this diversity is beginning to be represented with the increasing publication of SfD case studies. However, many of these case studies are focused on specific SfD programmes, and they vary in the extent to which they portray a deep understanding of the contexts in which these programmes are delivered. 
As a result, instrumental perspectives focused on SfD programmes remain very prominent within the literature and are particularly influential in debates regarding the evidence on $\mathrm{SfD}$. To counterbalance this, our collective aspiration and approach within this book is somewhat different: as previously discussed, rather than seeking to develop universal, generalizable understandings of $\mathrm{SfD}$, we hope instead to offer a 'wide-lens' perspective which captures the very varied, specific contexts within which community-level SfD activities and experiences can be understood.

\section{SfD evidence, evaluation and research}

Within SfD there is extensive and intense debate amongst stakeholders about the movement's 'evidence-base', with widespread concern expressed about a lack of credible evidence regarding the processes and - especially - the impacts of SfD programmes. These concerns mirror those found more broadly in international development, where calls for evidence-based policy have been conflated with increasing requirements for results-based accountability to Northern donors (Taylor, 2013). Thus, as the SDPIWG observed in 2006:

The absence of a strong body of compelling evidence in support of Sport for Development and Peace is repeatedly identified as a barrier to convincing policy makers and private sector donors to increase support for Sport for Development and Peace. (SDPIWG, 2006: 75)

More recent commentaries suggest that this issue has not been resolved despite the burgeoning of SfD scholarship in the interim. Five years after the SDPIWG's observation, Jay Coakley, one of the contributing authors to the 2007 Toronto Literature reviews on SDP, suggested that far from being evidence-based:

Sport-related decisions and policies remain shaped primarily by unquestioned beliefs grounded in wishful thinking, the idealized testimonials of current and former athletes, and the hunches of sport scientists. (Coakley, 2011: 307)

In a similar vein - and notwithstanding the publication of some substantial works, including 'Harnessing the Power of Sport for Development and Peace' (SDPIWG, 2008a) - Cornelissen was driven to comment that:

One of the biggest problems with the sport for development movement is the lack of an evidentiary base and the often substantial gap between theory and practice. (Cornelissen, 2011: 507) 
As the critiques offered by Coakley (2011) and Cornelissen (2011) show, the increased attention given to 'evidencing' SfD is partly a consequence of the issues at stake being positioned at an intersection of SfD scholarship, policy and practice. The UN, for example, has advocated that academia should make a contribution to SfD through:

build[ing] a strong evidence-base for the effective and efficient use of sport-fordevelopment and peace that can feed into the development of viable policy recommendations. (UN, 2012: 21)

Two themes consistently recur in these critiques. The first of these, particularly evident in the statement by the SDPIWG (2006), is that stronger evidence about the impact of SfD is necessary as a tool for advocacy that would help persuade relevant stakeholders to support and invest in the sector. For example, the opening editorial of the Journal of Sport for Development spoke of the journal's desire to address the need for $\mathrm{SfD}$ to 'establish adequate evidence to ... survive broad contractions in foreign aid budgets' (Richards et al., 2013: 2). Similarly, the Danish Network for Sport and Development (2009: 5) identified continuing 'pressure and interest to effectively measure and demonstrate the outcomes and impacts of sports programmes [in order to contribute to] the process of legitimizing sport-for-development as a field'. The second theme concerns the call for improved evidence in order to enhance SfD policies and practice, evident in the above extracts from Cornelissen (2011), Coakley (2011) and the UN (2012). The argument is that more reliable data is needed about how successful SfD programmes operate and what impacts they achieve, to allow policymakers and practitioners to learn from others' practice and design and deliver their own initiatives more effectively.

While it is not the intention here to provide a comprehensive review of the evidence now available on $\mathrm{SfD}$, it is pertinent to recognize Darnell's important contribution:

Given the different meanings of sport, the situated politics of development and the social complexities of sport and development, respectively, the idea that practitioners, scholars or activists will ever know with certainty whether, where or how sport is positive or effective for meeting development goals is unrealistic and unreasonable. (Darnell, 2012: 23)

Darnell's caveat is relevant to the debates considered throughout the remainder of the section and yet it is one that is infrequently acknowledged. Rather, across different stakeholders in $\mathrm{SfD}$, concerns have commonly centred on the scale and, especially, quality of the SfD evidence-base. Cronin's (2011: 5) mapping of 
research into the impact of SfD revealed an impressive level of activity between 2005 and 2010, identifying 267 evaluation reports and academic sources and concluding that 'significant investment has been made ... in developing an "evidence-base" to demonstrate the impact of sport'. However, despite more evidence being available on SfD, the contributions of Coakley (2011) and others indicate significant unresolved concerns regarding its quality. The remainder of the section, therefore, explores the nature of the evidence on SfD that is currently available and the processes by which this evidence has been generated. The section first considers the approaches underpinning two forms of evidence building that have been instigated by SfD organizations and stakeholders 'reviews', focused on identifying, collating and assessing already available evidence, and monitoring and evaluation studies. It then examines the commonly close alignment between research and evaluation in SfD, and considers critiques based on alternative theoretical and methodological approaches. The discussion raises a number of questions about the perceived limitations of SfD evidence, ranging from practical matters of research design and implementation to more fundamental ones about the nature of evidence. Particular attention is paid to the epistemological and ethical issues raised when processes of knowledge production are defined and controlled by outsiders.

Reviews of the evidence available on SfD have taken place at different stages in the development of the movement. As mentioned earlier, in 2006 the SDPIWG commissioned a research team at the University of Toronto to review evidence on the contribution of sport to a variety of potential individual and social outcomes. Edited by Bruce Kidd and Peter Donnelly, these literature reviews (SDPIWG, 2007) were supplemented by the sound knowledge of various subject-matter experts' (SDPIWG, 2008a: viii) and provided the basis of the subsequent report, 'Harnessing the Power of Sport for Development and Peace' (SDPIWG, 2008a). This report, and the reviews that preceded it, were described as 'fulfil[ling] the mandate of the SDPIWG to deliver comprehensive policy recommendations to national governments' (ibid.: vii). Subsequently, a further evidence-based analysis was commissioned by the Commonwealth Secretariat to fulfil a similar purpose of 'providing support for Commonwealth governments and other key stakeholders seeking to strengthen the contribution of sport to development and peace work' (Kay and Dudfield, 2013: 1). Compared with these two reviews, a third that was commissioned by UK-based charity Comic Relief in 2011 had a somewhat different purpose: to 'map the research looking at the impact of Sport for Development programmes since 2005 by a selected group of organizations in the UK and internationally' (Cronin, 2011: 5). 
Cronin's review was undertaken to improve understanding of the evidencebase and to establish a 'baseline' whereby potential research gaps, overlaps and opportunities for collaboration could be identified.

A number of issues arise from these documents. All three reports demonstrate an alignment with a narrative review approach, a 'traditional' method of research synthesis in the social sciences (Weed, 2005: 79). However, the reports do not provide significant detail on their review strategy. Cronin's (2011: 8) report of the mapping exercise is the most informative about its processes, which involved searches of the ISI Web of Science, Google Scholar and multiple SfD 'knowledge bases'. Additional sources identified or offered by expert contributors were also included. While all three reports focus on peer-reviewed research and highlight the importance of quality standards, none adopted specific methodological approaches, such as systematic reviews or realist synthesis, that respectively place greater importance on assessments of the methodological quality or (contextual) relevance of evidence sources. As such, the reviews are themselves open to criticism for their lack of rigour.

Although the three reports lack formal mechanisms for assessing the methodological quality of the sources they draw on, they are all nonetheless critical of the evidence they review. Each therefore carefully qualifies its recommendations: Kidd and Donnelly, for example, express a high degree of confidence in the data available to them in four of the five themes addressed in their review, yet also point out that:

It is usual in such 'gold standard' research to present findings and conclusions in a tentative and cautious way, without making grand claims about 'causality'. Such caution is appropriate, and the research team has been even more cautious in its overall conclusions because of the nature of the data. (Kidd and Donnelly, 2007: 3)

Yet despite expressed reservations of this type, all three reviews draw largely positive conclusions about the potential impact of sport on a variety of development outcomes. This means they effectively made 'the case for sport' that their commissioning agencies sought. The international standing of these commissioning agencies has in turn accorded the reports a significant status, which has potentially made them influential components of the SfD 'evidence-base'.

Further caveats attach to the potential influence of the reviews when the contextual relevance of their evidence is considered. All three reviews draw on evidence from both the global North and South but rarely separate them, and none directly confront the question of the geographical and cultural relevance 
of individual studies. The distinction between 'Northern' and 'Southern' sources is acknowledged by Kidd and Donnelly (2007) and SDPIWG (2008a), but this differentiation is not a significant feature of their reporting or analysis. Similarly, Kay and Dudfield (2013) draw on sources from the global North and South to identify which Commonwealth development goals have evidence-bases that support the use of sport, but do not address how this may apply differentially to different member countries within the economically and culturally diverse Commonwealth.

Cronin's (2011) report does provide useful context for this issue, by quantifying the geographical distribution of the evidence that she identified according to the country of authorship, and also the country in which the research was conducted. Her survey of authors for 2005-10 showed a preponderance of researchers resident in the global North (41\% Europe, 38\% USA, 7\% Canada), most of whose output related to sport and development in Europe (29\%) and North America (25\%), but who also wrote extensively on other regions - including Africa (20\%), the Middle East (6\%) and Asia (3\%). Most of the evidence available on sport and development was therefore about the use of sport in high-income countries, and most of the evidence on middle- and low-income countries was compiled by researchers from the global North. Thus, while Coalter (2013: 174) argues that those claiming a lack of evidence ignore the existing 'large body of research evidence about personal and social development' through sport, the cultural appropriateness of much of this evidence (and the reviews undertaken) on $\mathrm{SfD}$ remains open to question.

The issue of cultural appropriateness also arises in relation to evidence gained through monitoring and evaluation studies. Monitoring and evaluation has been a primary focus in much of the overall debate regarding the SfD evidence-base, with particular concerns relating to the importance, and challenges, of measuring 'impact'. The expansion of the SfD sector has been accompanied by a rise in monitoring and evaluation of $\mathrm{SfD}$ programmes, as indicated by the 'proliferation of overlapping/competing toolkits' (Cronin, 2011: 22) designed to support the monitoring and evaluation of SfD. Cronin identifies the development, between 2003 and 2011, of 15 such toolkits - described as a 'reasonable number ... for a relatively small sector' (2011: 22). Empirical studies by academics of specific SfD programmes have also frequently been undertaken in the context of monitoring and evaluation exercises. Indeed, debates regarding the $\mathrm{SfD}$ evidence-base in academic literature have often focused (explicitly or implicitly) on monitoring and evaluation (e.g. Donnelly et al., 2011; Coalter, 2013), which in many contributions are not differentiated from other forms of research. 
In seeking to address limitations of the SfD evidence-base, policymakers, practitioners and academics have all called for improved measurement of impact through monitoring and evaluation. Often, such contributions have been aligned with concerns about the 'unscientific' and potentially biased nature of what has been available:

With little more than anecdotal evidence, beliefs about the impact of sport in development are driven mainly by heartfelt narratives, evocative images, and quotable sound bites of individual and community transformation, packaged and delivered more often than not by those running the programs. (Hartmann and Kwauk, 2011: 285-286)

Directing their criticisms especially at so-called 'anecdotal' evidence, Hartmann and Kwauk (2011) suggest that the problems affecting current production and use of evidence are primarily the outcome of poor - qualitative - methodology. Elsewhere, calls to improve the quality of evidence generally align with positivist methodology and quantitative methods. For example, Akindes and Kirwan (2009: 219) call for 'objective analysis of the impact of current programmes', and Schulenkorf (2012: 9) for 'key performance indicators [that] would allow for a more rigorous evaluation of impacts'. Likewise, in relation to both measurement of impact and methodologies to do so, Cronin (2011) advocates the use of baseline data, 'strong behavioural indicators', validated data collection instruments, and 'controlled trials'. All of these contributions highlight limitations of existing approaches to measuring impact, as does Coalter (Coalter, 2010c: 100), who criticizes SfD monitoring and evaluation for its 'degree of uncertainty about valid impact measures'.

Coalter's (2010b, 2013, 2014) particularly prominent and increasingly strongly voiced contributions to the debates regarding SfD evidence do, however, contain some inconsistencies. On the one hand, he argues for an approach that places empirical pragmatism before issues of epistemology (2013). On the other hand, in a further contribution, he does not appear to baulk at recognition of the positivist underpinning of his and others' attempts to measure SfD impact (Coalter, 2014). More generally, and across many of his publications, Coalter $(2007 a, 2013,2014)$ promotes approaches to evaluation based on a realist epistemology. In citing Pawson and colleagues, whose work has underpinned the development of realist evaluation (e.g. Pawson and Tilley, 1997; Pawson, 2006), Coalter draws valuable attention to the importance of considering the mechanisms or processes that may contribute to achieving outcomes. However, the central importance that Pawson gives to the differential influences of varied 
contexts is not apparent in Coalter's (2013) key conceptual and empirical contributions. Furthermore, his emphasis on identifying a 'sequence of causes and presumed effects' (emphasis added; Coalter, 2009: 68) is more representative of logic models, as distinct from the programme theories which are central to Pawson's realist evaluation methods.

The use of logic models has been subject to significant criticism in other development sectors in which they have been utilized (Prinsen and Nijhof, 2015). Within SfD, Levermore (2011: 341) argues that logic models represent a 'rigid mentality to evaluation' and emphasize quantitative outcome measures that fail to capture important elements of personal and social development. Importantly, critiques of logic models also connect to wider issues related to the production of knowledge on SfD. Levermore (ibid.) further states that the 'wider and deeper epistemology of the programme is left unevaluated' through the 'top-down' application of logic models on behalf of donors who determine the scope of evaluation and the objectives measured and, as a result, ignore the views of programme 'recipients'. Kay (2012) has similarly voiced concerns about the prominence of such methods in SfD, and has highlighted the confusion and ambiguity that the method can cause, especially as different donor agencies use terms in different ways (and commonly require separate reports). She quotes Win's commentary on the experience of being trained to use the method:

We have to constantly remember what a goal, purpose, objective or output means to each one of you. We have learned painfully that all of these terms do not mean the same to everyone. The whole thing has been reduced to a farce. We laugh in workshops when you Northerners aren't there. 'Is that a goal? No, maybe it's an output!' (Win, 2004: 126)

Kay argues that, as a result, many monitoring and evaluation systems impose substantial workloads for little apparent return and through their day-to-day irrelevance come to be viewed solely as mechanisms for accountability to funding agencies. Win argues that this reduces rather than enhances learning, and also discourages transparency and openness: 'I am too scared to talk too much, just in case I say the wrong things. I withhold information that might damage my organization' (Win, 2004: 126). Kay further questions the cultural appropriateness of approaches which adopt rigid, formulaic reporting mechanisms that limit the level of detail and richness that can be captured. She cites Win's further comment:

We spent three days trying to fit visions, objectives, strategies and our way of seeing the world into the differently shaped blue, green and yellow cards. It was 
really not funny, though. It was painful. Nobody understood the method and the logic behind it. It did not make sense for many of us who are Ndebele- or Shona-speaking. In our language we express ourselves in paragraphs, not in short phrases or sentences. We are an oral people. We don't think in boxes either. (Win, 2004: 124)

The issue of insider-outsider production of evidence is also raised by authors concerned that the direct involvement of SfD practitioners in the production of knowledge is a further weakness that undermines the quality of the SfD evidence-base (Cronin, 2011; Donnelly et al., 2011; Hartmann and Kwauk, 2011). Cronin (2011) suggests that participation in and ownership of research by SfD organizations may compromise the 'scientific quality' of research, while Donnelly et al. (2011) consider that the majority of SfD NGOs lack the skills required to undertake 'scientific' monitoring and evaluation. These weaknesses are considered to lead to the production of overly simplistic numeric data and to encourage the use of narrative accounts that appear designed more to support advocacy efforts on behalf of SfD than to evaluate it. Hartmann and Kwauk's (2011) critique is but one of a number contributions to decry the production of anecdotes and/or 'heartfelt narratives' in support of SfD (e.g. Schulenkorf, 2012; Coalter, 2013; Sanders et al., 2014).

The above critiques of the SfD evidence-base align with broader themes in the SfD literature. The positivist orientation that underpins the 'lack of evidence' narrative is logically connected to the wider 'instrumental' strand within the SfD literature. This trend is compounded by a prevalent tendency to conflate evaluation, as a specific methodology, and research. The many SfD case studies that are tightly focused on specific programmes (e.g. Bennett, 2012; Weinberg and Rockenfeller, 2012; Gannett et al., 2014; Richards and Foster, 2014; Siefken et al., 2014), further contribute to the distinction between evaluation and wider forms of social research being underplayed. The specific focus and methodologies of narrowly focused SfD research often arise from the constraints of research funding which have commonly been associated with particular programmes. In our own studies, some of which have been similarly funded and shaped by SfD donors, we adopt an epistemological position that differs considerably from the instrumental strand within SfD literature, as we discuss further below.

Reflecting broader paradigmatic arguments, Darnell (2012: 4) warns that instrumental approaches to research and evaluation may 'fail to challenge the relations of power, privilege and dominance' within and beyond SfD. A number of other authors also raise similar concerns about the types of research and evaluation that are frequently promoted to overcome the weaknesses in the SfD 
evidence-base. They argue that particular forms of knowledge and knowledge producers tend to be privileged (e.g. Nicholls et al., 2011; Kay, 2012), which results in the subjugation of the views of those directly involved in SfD activities in local communities within the global South. Such critiques have been made most strongly, but not solely, by authors who are more broadly aligned with the 'critical-theoretical' strand within SfD research, which explores the maintenance of existing (global) relations of power and the depoliticization of development policies and practices (Mosse, 2004). Authors within this strand commonly utilize critical theories to frame their analysis, in contrast to the approach within instrumental SfD literature, which instead aims to propose new theories for SfD. However, while Darnell and Hayhurst (2012: 115) identify that theoretical frameworks which have been applied to $\mathrm{SfD}$, such as Gramscian hegemony, could 'lend insights into local struggles and negotiations for development', much of the SfD literature in critical-theoretical tradition remains internationalist in focus and methodology. This is itself reflective of the prevalence of academics from the global North in both shaping enquiry and documenting understandings of $\mathrm{SfD}$ research.

Within the development studies field, Mosse (2004: 641) recognizes that neither instrumental nor critical-theoretical perspectives do 'justice to the complexity of policy-making and its relationship to project practice, or to the creativity and skill involved in negotiating development. With the exception of a few notable counterexamples (e.g. Guest, 2009; Hasselgård and Straume, 2015), this critique could be collectively applied to the emerging field of SfD literature. For different reasons, within both the instrumental and critical-theoretical strands, the value of research designs that give prominence to qualitative narratives collected from a variety of local stakeholders has been underplayed. Critical theorists do argue for postcolonial approaches that involve examination of local resistance to hegemonic power (Darnell and Hayhurst, 2012, 2014). However, this does not necessarily foreground local perspectives but may instead constitute them within an overall analytic narrative. Even more pernicious are the arguments that are presented in the instrumental strand of SfD literature, where the credibility and importance attached to supposedly 'objective' assessments of $\mathrm{SfD}$, not only devalues the subjective perspectives of those involved in $\mathrm{SfD}$, but appears to dismiss anyone speaking of $\mathrm{SfD}$ with particular subjectivities as guilty of offering an 'anecdotal' narrative that has no value. While the wide use of anecdotes in support of SfD is unquestionable, the tendency for criticisms of anecdotes to be extended to qualitative data that is rigorously collected and systematically analysed is worrying and uninformed. It is 
notable that the distinction between promotional testimonies and research data is not addressed in critiques offered by those with an instrumental or positivist orientation to SfD research and evaluation. Without such a distinction, all local voices, and the knowledge and detail (only) they can provide, risk being ignored or lost in the ill-conceived quest to build a 'scientific' evidence-base for SfD.

\section{Localizing sport for development in Zambia: our research approach}

The previous sections have raised questions about the extent to which research approaches to $\mathrm{SfD}$ to date can provide understanding of its complexities and nuances. This is not to question the validity of published $\mathrm{SfD}$ research findings but rather to recognize, as Mwaanga and Mwansa (2014) do, the extent to which $\mathrm{SfD}$ research and researchers have so far offered only limited opportunities for stakeholders from outside the global North to express their perspectives of SfD. In part, this reflects the practical difficulties of undertaking such research when greater resources to do so reside in the global North and are often directed towards relatively short-term, programme-focused evaluations. Additionally, there are more fundamental epistemological and methodological challenges to producing decolonized knowledge of development, as discussed in McEwan's (2009) important book and considered where relevant below.

The limitations and challenges discussed above, that characterize much existing SfD research, have prompted advocacy for alternative approaches. Suggestions include the use of ethnographic or anthropological methodologies (Hayhurst, 2009; Darnell, 2012) and the development of local ownership of and active participation in SfD research (Kay, 2009; Njelesani, 2011; Spaaij, 2012). Such approaches have been utilized in specific, but relatively isolated, studies. Contributions by Armstrong (2004), Guest (2009), Fokwang (2009), and Kath and van Buuren (2014), to give but four examples, stand amongst those that have used different methodological approaches to present locally grounded accounts of SfD in different African contexts. It is notable that these authors have varied backgrounds in relation to the location in which their research has been undertaken; nonetheless, all have spent considerable time within these localities. In situating their qualitative accounts within these locations, these authors also recognize, to differing extents, the ways in which $\mathrm{SfD}$ is influenced by local social, economic and political contexts. What is also common across these contributions is that they demonstrate, implicitly or explicitly, the potential for diverse forms of social research, as distinct from 
evaluation focused on evidence building, to provide increased knowledge and understanding of SfD in forms that are also valuable for informing policy or practice.

These broader trends in the SfD literature and research have bearing on the research we draw on in this book. Over the course of our studies in Zambia, our desire to address and counter problems associated with the prominence in SfD literature of instrumental and critical-theoretical strands has become more explicit, motivated by a desire to enhance understanding of SfD. In order to do so, we have increasingly turned to development studies literature. Methodologically, we have found ourselves increasingly aligned with the actor-oriented sociology of development advocated by Norman Long (2001). The genesis of Long's own actor-oriented approach derived from his frustration with the 'theoretical and methodological shortcomings of existing structural and generic theories of development that espoused various forms of determinism, linearity and institutional hegemony' (Long, 2001: 1). This critique resonates with the foregoing discussion of the limitations of the instrumentalist strand in SfD literature, and while the 'impasse' that Long describes occurred in development studies in the mid-1980s, the continued citation of his actor-oriented approach confirms its enduring currency and, by association, its potential value to SfD research. It is therefore through examination of aspects of the actor-orientated approach that we can explore the facets, strengths and limitations of our own research and, subsequently, the relevance of its findings and conclusions.

An actor-oriented approach is consistent with the philosophical assumptions of social constructionism. As such, we are principally concerned with 'understanding the processes by which specific actors and networks of actors engage with and thus co-produce their own (inter)personal and collective social worlds' (Long, 2001: 3). This is in accordance with both Darnell (2010a) and, especially, Nicholls et al. (2011), who advocate the importance of understanding the perspectives of those at the 'front line' and 'grass roots' of SfD practices. As well as agreeing with these authors, we additionally take from Long (2001) the recognition that 'development' also encompasses a multiplicity of actors, even at a local level, who may hold diverse positions and perspectives. As such, one of the characteristics of our research is what we think of as its 'wide-lens' approach, which encompasses perspectives of a particularly diverse range of Zambian actors associated with SfD. Collectively, these research participants are drawn from a variety of contexts with which SfD is connected, and from multiple levels within them; they include participants, peer leaders, community members, NGO staff, governmental stakeholders and international donors. ${ }^{4}$ Engagement 
with this array of SfD stakeholders has been made possible as a result of our engagement across various research projects, each of which has defined our relationships with research participants in particular ways (McEwan, 2009). Inevitably, the parameters and constraints of particular research projects have limited the extent to which we have been able to listen to voices that could further enhance understandings of SfD. This is particularly the case in respect to individuals, such as parents and young people not participating in sport, who may not have as direct an association with $\mathrm{SfD}$ as those who have more commonly been participants in our research (Long, 2001).

Irrespective of these particular limitations, it is hopefully apparent by this point that our alignment with an actor-oriented approach entails recognition of 'social heterogeneity' and 'differential interpretations' (Long, 2001: 240). Moreover, this approach requires similar thinking as to how we consider the practice of SfD. Long (2001: 32) steers us away from thinking of any development programmes as a "project" with sharp boundaries in time and space as defined by institutional apparatus of the state or implementing agency'. We must nonetheless acknowledge that a number of the studies that inform this book have been closely linked with specific SfD programmes, and that these programmes have to varying degrees been defined by funding from Northern agencies. This clearly has implications for attempts to decolonize SfD knowledge (McEwan, 2009), to which we return in the concluding chapter of the book. Furthermore, in terms of analysis of the historical dimensions of development interventions called for by Long, we can recognize some of our specific research projects as being subject to Coalter's (2013) broader critique of the cross-sectional nature of much SfD research. Still, what our collective analysis of the various data collected since 2006 offers is a broader examination of the various ways in which sport has been employed, with varying degrees of intentionality, for the purposes of contributing to social change, variously defined. This exploration of the ways in which sport is planned and delivered is complemented by our examination, in Chapter 6 in particular, of the place and influence of sport in the lives of young Zambians. In presenting these various perspectives, we seek to contribute to the 'de-reification' of both 'sport' and 'development' (Coalter, 2013).

The aspiration of this book, captured in its title, is to 'localize global sport for development.' Therefore, it is useful to also explore our understanding of, and approach towards, 'the local. The research that we present has been undertaken within Zambian communities, predominantly within Lusaka. We have spent time in these communities on a recurrent basis across a number of research projects and have gained considerable data from those who live and 
work within them. As outsiders to these localized contexts, however, we have to recognize that the understandings that we present in this book remain necessarily limited and provisional (McEwan, 2009). Furthermore, we do not seek to essentialize the particular communities in which we have undertaken research as being representative either of other local settings of SfD interventions or indeed of other Zambian communities; in particular, we have undertaken little research in the rural parts of the country. Long's (2001: 50) actor-oriented approach recognizes, however, that 'social action and interpretation are contextspecific and contextually generated'. All of our data collection has sought to gain a greater appreciation of the various contexts within which our Zambian research participants reside and within which SfD operates and occurs. Exploration of these contexts appears within specific chapters and is more generally infused throughout the book.

Our methodological approach has also involved examination of how local perspectives on SfD are linked to, and potentially influenced by, broader contexts. As the book's title suggests, our analysis seeks to examine the relationships between our locally oriented data and the global dimensions of SfD we have identified earlier in this chapter. However, as Lund (2010), Darnell and Hayhurst (2012), and Long (2001) himself warn, it is important to view the relationships between the local and global as multilayered rather than representing a simple dichotomy between the 'local' and 'global'. Moreover, it is important to avoid viewing $\mathrm{SfD}$, either in local or global terms, as being hermetically sealed from broader influences, such as those associated with development efforts more generally. Tracing the linkages between multilayered influences on SfD has also been a feature of research by Hasselgård and Straume (2015) involving locally collected data from Zimbabwe. These authors have adopted an approach that is broadly aligned with top-down models of policy implementation in examining local responses to Norwegian-funded programmes. Our methodology complements this but differs in starting with local actors' perspectives and considering how various multilayered aspects of the contexts in which they reside are reflected and refracted in these perspectives and their actions.

This bottom-up, multilayered approach is in line with the broader ontological, epistemological and theoretical orientation of Long's (2001) approach. He emphasizes the need to 'develop theory from below' rather than basing interpretation on theories that have a high level of abstraction and, as a result, tend to lead to reification of aspects of development processes. To put it differently, instead of utilizing theory as an 'explanatory framework', Long advocates an alternative 'type of theoretical construct [as a] set of conceptual tools which, 
rather than telling us anything substantive about the social world, suggests ways of approaching it' (Lund, 2010: 26). We have not subscribed to a singular explanatory theoretical framework in this research, especially as to do so may be to circumscribe what we hope is a holistic approach to analysis that integrates consideration of various dimensions of SfD in Zambia. Nevertheless, we seek to avoid the dangers of 'descriptive particularism' identified by Lund (2010) by utilizing various other conceptual and empirical literatures throughout the book. This is also done in recognition of the importance of appreciating the influence on actors of structures which Long characterizes as:

An extremely fluid set of emergent properties that, on the one hand, are a product of the interlocking and/or the distantiation of various actors' projects, while on the other, they constitute an important set of reference points and constraining/enabling possibilities that feed into further elaboration, negotiation and confrontation of actors' projects (Long, 2001: 62)

Our consideration of the relationships between the perspectives of Zambian actors and themes identified in broader literatures allows us to explore ways in which local agency may be shaped and constrained. In doing so, we endeavour to tread a fine balance between exploring the potential relevance of our findings to other settings and remembering that 'different social forms develop under the same or similar structural circumstances' (Long, 2001: 20).

At the close of this exposition of our methodology, a few pertinent issues remain. Our overall approach to this research is one that recognizes complexity in contexts, interventions, lives and the social world more generally. We cannot hope to capture the full extent of this complexity in this book, especially as our research has often been subject to pragmatic constraints in both design and implementation that Coalter (2013) recognizes are inevitable within SfD research. More fundamentally, we acknowledge and hope that we continue to be reflexive regarding our own positions and relationships with those involved in our research. This is not necessarily as simplistic as recognizing our position as privileged outsiders - and Davies' status, as a Zambian who has lived overseas for a number of years while working for Northern universities, may be particularly characterized by complexity. Nevertheless, recognition of our positions in respect to research participants has led to the development and use of specific techniques to counter as far as possible the power imbalances that have inevitably inscribed all of our data collection activities (McEwan, 2009). On a more long-term basis, we have sought to involve representatives of Zambian SfD organizations in the design and implementation of the research to different 
extents and in different ways. The adoption of this approach has not been to necessarily contribute to the improvement of specific programmes in the direct way that Coalter (2013) suggests. Rather, we hope that it has been one of mutual learning and personal and organizational development that may have value in contributing towards future progression. On our part, our ongoing conversations and interactions with those who have worked with us through the research have unquestionably improved our knowledge and hopefully made us more nuanced and reflective in our analysis. Irrespective of the validity (or not) of these interpretations, our position in being able to present them in this book remains a privileged and, therefore, problematic one (McEwan, 2009). We shall return to such issues in the concluding chapter as we consider the potential value and limitations of our methodological approach and research. Readers can, we hope, make something of their own assessment through considering our accounts in the intervening chapters.

\section{Notes}

1 The terms global North and global South are the ones that we have chosen throughout the book to collectively represent the respective distinction between higher- and lower-income countries. We recognize that this terminology can be contentious as multiple layers of complexity are subsumed by a geographical dichotomy which associates numerous diverse countries in two overarching categories. Nevertheless, we use these terminologies as the divide between global North and South is still important in shaping development thinking (McEwan, 2009; Williams et al., 2014) and, we would argue, SfD. In making references to the global North or South throughout the book, we have been careful to avoid essentializing either.

2 The terminology and acronym used here, 'sport for development and peace' (SDP), is commonly used and is also the terminology adopted by the UN. The issue of 'peace' is not one that has been significantly referred to relation to Zambian practice and neither has it been a focus of our research. As such, the alternative terminology of 'sport for development' (SfD) is used in reference to our research, although we do replicate the terms used in other sources where we reference them.

3 It is noted that Giulianotti (2011a) locates the entire SfD sector within 'global civil society'. Importantly, appending 'global' differentiates the concept to which Giulianotti refers from definitions which identify civil society as comprising a range of organizations and other agencies that are independent of government and are commonly understood to be not for profit.

4 Throughout the book, we preserve the anonymity of our interviewees but identify their role and status where presenting direct quotations from interviews. For adult interviewees, we also note the type of organization that they represent. Both the gender and home community within Lusaka of young people who are quoted are provided. 